\title{
Predictive Control of an Asymmetric Multicell Converter with Floating Cells.
}

\author{
Ricardo Aguilera, Daniel Quevedo \\ School of Electrical Engineering and Computer Science \\ The University of Newcastle, Australia \\ Email: aguilera.ricardo@gmail.com,dquevedo@ieee.org
}

\author{
Pablo Lezana \\ Departamento de Ingeniería Eléctrica \\ Universidad Técnica Federico Santa María, Chile \\ Email: pablo.lezana@usm.cl
}

\begin{abstract}
In this work we present an Asymmetric Multicell Converter fed by a single DC source per phase. The proposed control method is divided in two parts. Firstly, the main cells are handled by using a fundamental frequency switching pattern. In parallel, a Model Predictive strategy is used to control the remaining cells which use floating capacitor instead of a DC source. The target of this controller is not only to improve the output current quality, but also regulate the floating capacitor voltages of the small cells in order to achieve the desired number of levels of the output voltage. In this way, the main cells provide the active power to the load whereas the small cells provide reactive power to reduce the harmonic pollution produced by the main ones.
\end{abstract}

\section{INTRODUCTION}

Multilevel converters have arisen as an important technology to handle electrical power $[1,2]$, being widely used in different kind of applications such as AC motor drives [3], active filters [4] and uninterruptible power supplies [5]. One important multilevel architecture is the Multicell Cascade H-bridge (CHB) converter which has been under constant study in recent years [2]. In a standard k-cell CHB converter, $k$ isolated DC sources per phase with identical voltage levels are required to fed each cell. To do this, normally a multiple-winding transformer is required. The number of different levels in the output voltage will be $2 k+1$. It is important to note that in this case there are some redundant switch combinations which generate the same voltage level. Another way to increase the number of levels is to implement a so-called asymmetric CHB converter. This configuration considers $k$ isolated DC sources per phase with unequal voltage levels $[6,7]$. This allows one to take advantage of the redundance in order to obtain a maximum of $3^{k}$ different levels in the output voltage. Thereby, a smaller number of isolated DC source is required to obtain the same number of levels than in the symmetrical case.

To mitigate even more the problem of having multiple isolated DC source, in $[8,9]$ a single phase CHB converter fed by a single DC source has been presented. Since only one cell is electrically fed, the remaining $k-1$ cells utilize electrically floating capacitors to synthesize the required intermediate voltage levels. Here, a selective harmonic elimination (SHE) strategy is considered to produce a pulse width modulation (PWM) pattern to synthesize the output voltage. In principle, this technique presents an improvement in regard to the DC source requirement. However, using floating capacitors also in- creases the control complexity, due to the fact that intermediate voltages across the floating capacitors must be regulated precisely for correct operation of the converter. Here, the authors have proposed to use some redundant switch combinations to regulate the floating capacitor voltage. A disadvantage of doing this is that one cannot reach the maximum number of levels in the output voltage. Moreover, capacitor voltages are strongly affected by the load current and the time period in which redundant switch combinations are applied, making the regulation of the floating capacitor voltages a hard task. A specification of a stable operation zone for a single DC source CHB is presented in [10]. In this case all cells are controlled using a high frequency PWM. The definition of the stable area is based on the DC voltage ratio between DC voltage of the main cell and the floating capacitor voltage of the small cell and the current load level. In this way, it is possible to generate the maximum number of levels in the output voltage under certain conditions.

Using floating capacitor H-Bridge cells has been also studied for 3-phase applications. In [11], a control strategy for a neutral point clamped (NPC) along with a floating CHB has been presented. In this case, NPC inverter is used to supply the active power to the load while H-bridge cells provide reactive power to improve the load current quality. The output voltage is synthesized through a multicarrier PWM pattern. In addition, a predictive control strategy for floating capacitor voltage regulation has been proposed. This technique takes into account the fact that variations in the commonmode voltage allow one to transfer active power from the NPC converter to the floating cells. Consequently, redundances are not needed allowing us to obtain the maximum number of levels in the output voltage. Recently, a different control method for 3-phase CHB inverter using a single DC source per phase has been presented [12]. Here, a CHB comprised of 2-cells per phase is analyzed. The main cell, which provides the active power to the load is controlled by using a 3 levels fundamental frequency switching pattern. The error, between this pattern and the desired fundamental voltage is used as a reference for the floating cells. Here, the authors have shown that, if there are some redundances in the switch combinations, one can slightly shift the main cell voltage pattern to achieve the floating capacitor voltage regulation. In this case, the maximum voltage levels cannot be reached either. 
In the present work we present a Model Predictive Control strategy applied to a 3-phase CHB inverter fed by a single DC source per phase. To handle the main cells, a fundamental frequency switching pattern is used to synthesize their output voltages. In parallel, a Model Predictive strategy is used to control the remaining floating cells. With this aim, an inverterload model is presented which considers the converter switches as control inputs of the system. The key novelty of our proposal lies in the fact that the predictive controller directly provides the switch combination not only to synthesize the output voltage of the small cells to reduce the harmonic pollution in the output current, but also to regulate the floating capacitor voltages. Since no redundances are needed, our proposal allows the converter to generate the maximum number of levels in the output voltage. In addition, we will also show that pre-charge of the floating capacitor voltages is not compulsory.

\section{CASCADE H-BRIDGe CONVERTER}

The concept of Cascade H-Bridge converter (CHB) was introduced in $[13,14]$. The main idea is the series connection of voltage sources, called generically as cells. In most of the cases these cells are composed by an isolated dc-link voltage and an H-Bridge. Typically, the cells are fed with dc sources which have the same magnitud ( $v_{d c a 1}=v_{d c a 2}$ in Fig. 1(a)), then the converter is called Symmetric CHB (SCHB), whereas if $v_{d c a 1} \neq v_{d c a 2}$ the converter is called Asymmetric CHB (ACHB). The SCHB converters are highly modular and have many redundant states which can be used to spread the power among cells and improve the operation under fault. The ACHB converters sacrifice redundant states in order to increase the number of voltage levels in the output of the converter, and hence improve the quality of the output currents.

The output voltage of each H-bridge can be defined as:

$$
\begin{aligned}
v_{x i} & =\left(S_{x i 1}-S_{x i 2}\right) v_{d c i} & & x \in\{a, b, c\}, i \in\{1 \ldots k\} \\
& =S_{x i} v_{d c i} & & S_{x i} \in\{-1,0,1\} .
\end{aligned}
$$

Then the total output inverter voltage is:

$$
v_{x n}=\sum_{i=1}^{k} v_{x i} .
$$

Table I shows all the possible combinations for the cell and the total output voltage of a converter with two cells per phase $(k=2)$. For an SCHB $\left(v_{d c a 1}=v_{d c a 2}\right)$ the converter can reach up to 5 voltage levels, while for an $\mathrm{ACHB}\left(v_{d c a 1} \neq v_{d c a 2}\right)$ up to 9 levels can be reached if a 1:3 ratio between the dc voltages is used [15].

\section{A. Topology Description}

In high power applications the switching frequency is limited in order to reduce the loses and/or due to semiconductor restrictions. Low switching frequency leads to low order harmonic distortion, which affects the close-loop behavior and can generate torque pulsations if an electrical machine is

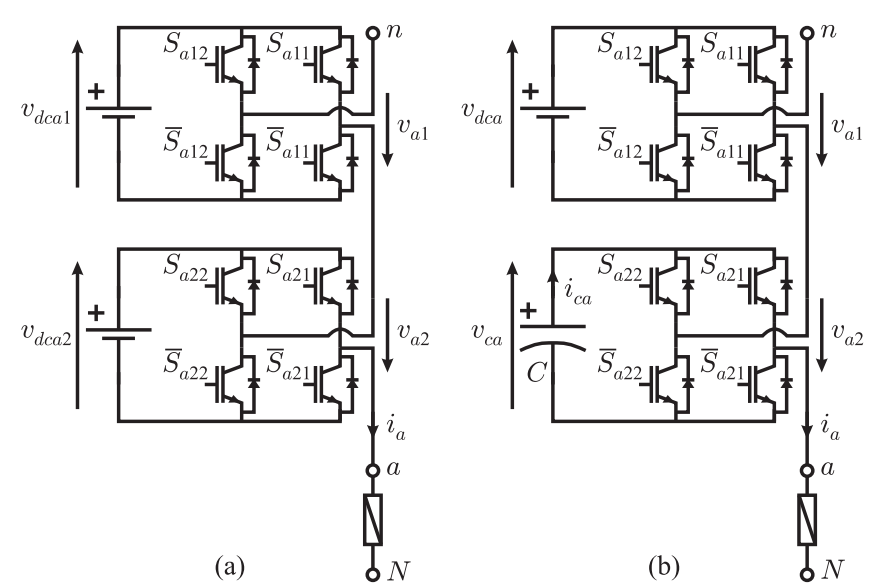

Fig. 1. Cascade H-Bridge topologies: (a) All cells fed with active voltage sources; (b) Proposed topology.

TABLE I

TOTAL OUTPUT VOLTAGES FOR TWO CELLS CONNECTED IN SERIES.

\begin{tabular}{|c|c|c|c|c|}
\hline$S_{a 1}$ & $S_{a 2}$ & $v_{d c a 1}$ & $v_{d c a 2}$ & $v_{a n}$ \\
\hline-1 & -1 & $-v_{d c}$ & $-v_{c a}$ & $-v_{d c}-v_{c a}$ \\
-1 & 0 & $-v_{d c}$ & 0 & $-v_{d c}$ \\
-1 & 1 & $-v_{d c}$ & $v_{c a}$ & $-v_{d c}+v_{c a}$ \\
0 & -1 & 0 & $-v_{c a}$ & $-v_{c a}$ \\
0 & 0 & 0 & 0 & 0 \\
0 & 1 & 0 & $v_{c a}$ & $v_{c a}$ \\
1 & -1 & $v_{d c}$ & $-v_{c a}$ & $v_{d c}-v_{c a}$ \\
1 & 0 & $v_{d c}$ & 0 & $v_{d c}$ \\
1 & 1 & $v_{d c}$ & $v_{c a}$ & $v_{d c}+v_{c a}$ \\
\hline
\end{tabular}

used as load. For such applications, the concept of CHB can be extended to active filter operation [8-12], by connecting a cell in series with a main converter. This main converter provides the active power to the load, while the cell is intended to supply only reactive power, mainly related with the low frequency harmonics generated by the main converter.

As only reactive power is ideally supplied by the cell, its average dc-link voltage tends to be constant, then the active DC source can be replaced by a electrically charged capacitor. In real systems however, a control scheme must be implemented in order to keep this voltage constant due to losses in parasitic resistors, load transients and also to guarantee a proper start-up of the overall system.

In this work a regular one-cell per phase CHB converter is considered as the main converter. A second cell fed by a floating capacitor is connected in series (as shown in Fig. 1(b)), which will act as an active filter.

\section{B. Converter Model}

According to (1) and (2), the total output voltage for the converter (Fig. 1(b)) is:

$$
v_{a n}(t)=S_{a 1} v_{d c a}+S_{a 2} v_{c a}(t)
$$

where the capacitor voltage $v_{c a}$ is defined by:

$$
\begin{aligned}
v_{c a}(t) & =v_{c a}(0)-\frac{1}{C} \int_{0}^{t} i_{c a} d \tau \\
& =v_{c a}(0)-\frac{1}{C} \int_{0}^{t} S_{a 2} i_{a} d \tau
\end{aligned}
$$


Similar equations can be derived for phases $b$ and $c$. Finally, the expression for the output currents are:

$$
\begin{aligned}
v_{a}-v_{N n} & =R i_{a}+L \frac{d i_{a}}{d t} \\
v_{b}-v_{N n} & =R i_{b}+L \frac{d i_{b}}{d t} \\
v_{c}-v_{N n} & =R i_{c}+L \frac{d i_{c}}{d t} \\
v_{N n} & =\frac{v_{a}+v_{b}+v_{c}}{3}
\end{aligned}
$$

\section{Model Predictive CONTROL}

Model Predictive Control (MPC) or receding horizon control (RHC) [16] is a control paradigm which calculates the control action by solving, at each sampling instant, an optimal control problem which forecasts, over a finite horizon, the future system behavior. To achieve this, the current system state is taking into account. This generates an optimal control sequence. Finally, the control action to be applied to the plant is the first element of this sequence. The main advantage of MPC is that constraints and non-linearities can be included. Nevertheless, a large amount of calculation is required to obtain the optimal control sequence.

A variation of this predictive strategy have emerged as a promising control technique for Power Converters, the socalled, Finite Control Set Model Predictive Control (FCSMPC) $[17,18]$. The main advantage of this predictive strategy comes from the fact that switching actions are expressly taking into account. Consequently, modulation stages (to handle the switching elements) are not required. This strategy directly considers the switching elements as the control input which can take only two values $s_{i}=1$ if the switch is closed and $s_{i}=0$ if the switch is open. This produces that the input control is restricted to belong to a finite set.

FCS-MPC strategy is implemented in discrete time with sampling frequency $f_{s}=h^{-1}$. Therefore it is necessary to obtain a discrete model of the plant

$$
x[k+1]=f(x[k], s[k]),
$$

where $x[k] \triangleq\left[x_{1}, \cdots, x_{n}\right]^{T}$ are the $n$ system states and $s[k] \triangleq\left[s_{1}, \cdots, s_{m}\right]^{T}$ are the $m$ control inputs, in this case the converter switches.

At each discrete instant $k$, a measurement of the system state $x[k]$ is taken. To forecast the system behavior, a cost function which considers the impact of the converter switches $s_{j}$ over the system error is evaluated. Using a Euclidean norm of the system error, the proposed cost function is expressed via:

$$
J[k](\vec{s}[k], x[k])=\sum_{l=k+1}^{k+N}\left\|x[l]-x^{\star}[l]\right\|_{P}^{2},
$$

where $\|\cdot\|_{P}$ stands for

$$
\left\|x[l]-x^{\star}[l]\right\|_{P}^{2} \triangleq\left(x[l]-x^{\star}[l]\right)^{T} P\left(x[l]-x^{\star}[l]\right),
$$

and

$$
P=\operatorname{diag}\left\{\lambda_{i}, \ldots, \lambda_{n}\right\}
$$

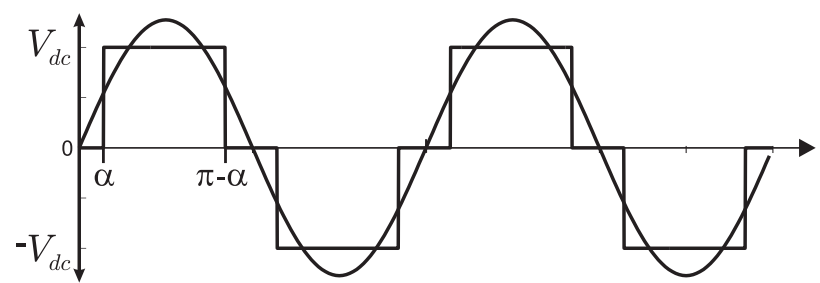

Fig. 2. Fundamental frequency PWM pattern for main cells.

Here, $x^{\star}$ stands for the desired system reference, while $\lambda_{i}$ are nonnegative scalar tuning parameters. The decision variables are:

$$
\vec{s}[k] \triangleq\{s[k+1], \cdots, s[k+N]\}
$$

The vector $\vec{s}[k]$, thus, contains tentative switching actions over a horizon of finite length $N$. The optimal switching action to be applied at time $k+1$, namely $s_{\text {opt }}[k]$ will be the first element of $\vec{s}[k]$. Then, at the next instant, $k+1$, the cost function $J[k+1](\vec{s}[k+1], x[k+1])$ is minimized using fresh state measurements. This gives $s_{\text {opt }}[k+1]$ and so on.

\section{Proposed Control Strategy}

As mentioned above, we have divided the control of the CHB converter in two parts. Firstly, we will consider a low fundamental frequency modulation to synthesize the output voltage of the main cells. In parallel, floating cells are controlled by an MPC strategy to improve the quality of the load current.

\section{A. Fundamental Frequency Pattern for main Cells}

Since the active power will be supplied by the main cells which are fed by an isolated DC source, a fundamental frequency PWM pattern is considered to handle these cells in order to reduce the power losses and commutation stresses of the switches. To do this, firstly we represent the desired sinusoidal reference per phase via:

$$
i_{x}^{\star}(t)=I^{\star} \sin \left(2 \pi f_{0} t+\phi_{x}\right),
$$

where $I^{\star}$ is the current amplitude reference, $f_{0}$ is the fundamental frequency and $\phi_{x}$ is the corresponding angle per phase.

In Fig. 2, a representation of the desired PWM patter for the main cells is presented. The amplitude of the fundamental component of this voltage can be expressed by

$$
V_{x i}^{1}=\frac{4 V_{d c}}{\pi} \cos (\alpha)
$$

where, $\alpha$ is the commutation angle.

Considering the amplitude of the load current reference $I^{\star}$ and the load parameters $R$ and $L$, angle $\alpha$ can be expressed via:

$$
\alpha=\cos ^{-1}\left(\frac{\pi V_{x i}^{1}}{4 V_{d c}} I^{\star} \sqrt{R^{2}+\left(2 \pi f_{o} L\right)^{2}}\right) .
$$

In this way, one can easily control the main cells by considering only the fundamental frequency behavior. 


\section{B. FCS-MPC of floating Cells}

Since floating cells must transfer reactive power to the load using a reduced DC voltage, one can select a higher commutation frequency to synthesize their required output voltage. However, an active control of the floating capacitor voltage is also needed. To overtake this complex task, we propose to use an FCS-MPC strategy to control these cells.

As already stated in Section III, firstly we need a discrete time model of the system. To do this, capacitor voltage equation (4) can be transformed into discrete time using forward Euler approximation. Therefore, this voltages are expressed by:

$$
v_{c x}[k+1]=v_{c x}[k]-\frac{h}{C} i_{x}[k] S_{x 2}[k],
$$

If we consider that capacitor voltages remain constant within a sampling period $h$, a more suitable way to obtain a discrete time model of the load current (5) is applying zero order hold approximation. Thus, load current can be represented by:

$$
i_{x}[k+1]=K_{a} i_{x}[k]+K_{b}\left(v_{a}[k]-v_{N n}[k]\right),
$$

where

$$
K_{a}=e^{-h \frac{R}{L}}, K_{b}=\left(1-K_{a}\right) / R,
$$

and $v_{N n}$ stands for the common-mode voltage which is expressed by:

$$
v_{N n}[k+1]=\frac{v_{a}[k]+v_{b}[k]+v_{c}[k]}{3} .
$$

Considering a prediction horizon of $N=1$ the proposed cost function (7) can be expressed via:

$$
J[k]=\sum_{x=a}^{c}\left(\left(i_{x}[k]-i_{x}^{\star}[k]\right)^{2}+\lambda\left(v_{c x}[k]-\frac{V_{d c}}{3}\right)^{2}\right) .
$$

Here, $\lambda$ is a weighting factor which allows one to have a trade off between load current quality and capacitor voltage regulation. It is important to notice that floating capacitor voltage reference is chosen as $V_{d c} / 3$. In this way, we can reduce the commutation stress of the switches in these cells and also we can generate the maximum number of levels in the total output voltage.

Finally, a block diagram of the proposed predictive method is presented in Fig. 3.

\section{RESUlTS}

Simulations of the proposed control scheme described in Section IV were carried out in a CHB converter with the parameters shown in Table II.

For comparison purposes, Fig. 4 shows the main variables of the system working without the floating cells. An current amplitude reference of $I_{x}^{\star}$ of 20 A was considered. As the main cell only commutates twice in a fundamental period, as shown in Fig. 4(b), the output current presents a high distortion level (Fig. 4(a)). Note however, that the current has slightly less

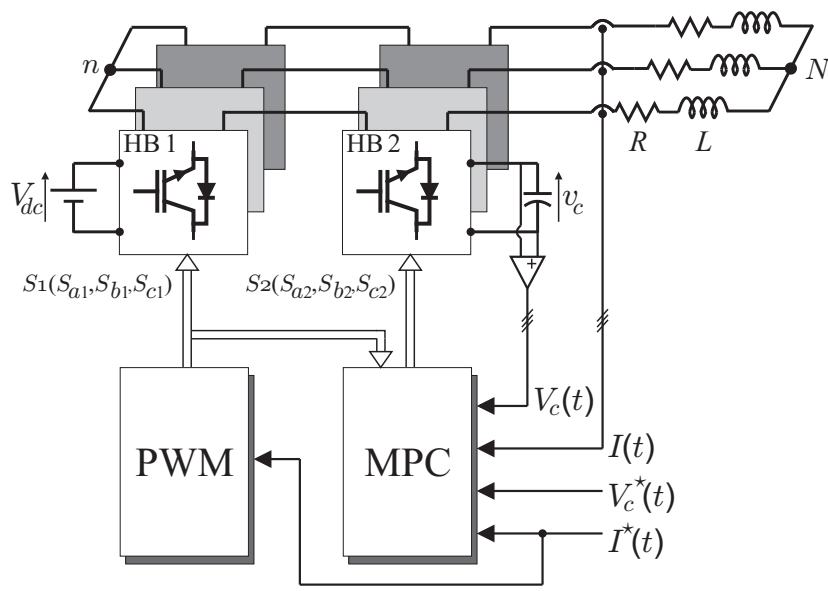

Fig. 3. Block Diagram of the proposed strategy.

TABLE II

SYSTEM MAIN PARAMETERS

\begin{tabular}{|c|c|l|}
\hline Symbol & Value & Variable \\
\hline$f_{s}$ & $5 \mathrm{kHz}$ & MPC sampling frequency \\
$C$ & $3.3 \mathrm{mF}$ & Floating dc-link capacitor \\
$L$ & $12 \mathrm{mH}$ & Load inductance \\
$R$ & $20 \Omega$ & Load resistance \\
$V_{d c}$ & $450 \mathrm{~V}$ & Main cell dc-link voltage \\
$v_{c x}^{*}$ & $150 \mathrm{~V}$ & Floating capacitor voltage reference \\
$\lambda$ & 1 & Weighting factor \\
\hline
\end{tabular}

harmonics than the inverter voltage, due to the phase voltage interaction through the load neutral point $N$, which generate the load voltage waveform shown in Fig. 4(c).

\section{A. Steady-State Performance}

Fig. 5 shows the performance of the proposed topology and control scheme under the same reference than Fig. 4.

It is clear that the current shown in Fig. 5(a) has a lower harmonic distortion. Indeed, only small spikes are observed in the currents. Note that those spikes occur when the main cell output voltage changes. At that instant the floating cells do
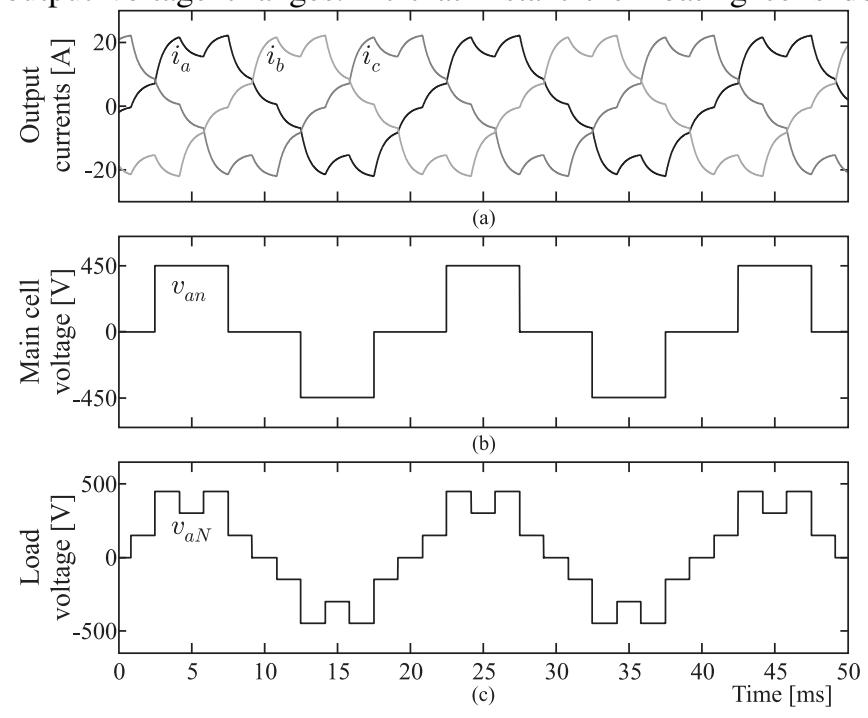

Fig. 4. Steady-state waveforms for the converter without the floating cell. 

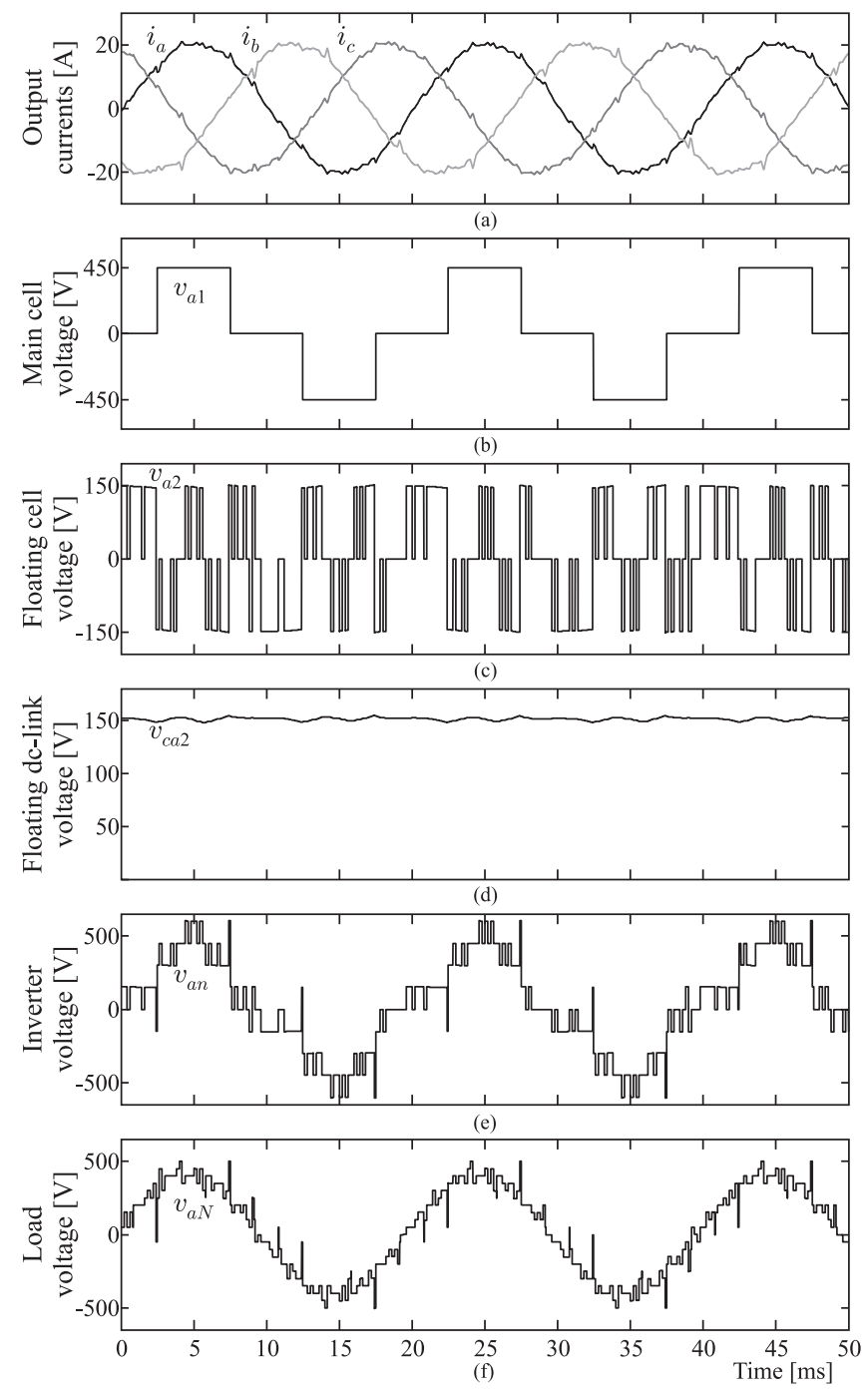

Fig. 5. Steady-state waveforms for the converter with the floating cell.

not have enough remaining actuation capability to override such large perturbation. Moreover, the main cell changes asynchronously compared to the MPC sampling, then the predictive control only notices this "perturbation" in the next sampling instant.

The voltage of a floating capacitor is shown in Fig. 5(d). This voltage is almost constant in comparison to its reference value. Then, the output voltage of the floating cell, shown in Fig. 5(c) corresponds to a 3-level waveform. Note that this voltage presents a wide spectrum due to the harmonic compensation and for the well-known pseudo-random switching pattern generated by FCS-MPC.

The inverter voltage shown in Fig. 5(e) has 9 levels due to the chosen ratio $v_{d c}: v_{c x}=3: 1$. In a similar way, see Fig. 4, the load voltage is clearly more sinusoidal, due to the interaction among the phase voltages.

Fig. 6 shows the current spectra for the converter working with and without the floating cell. These spectra confirm that the lowest-frequency/highest-magnitude harmonics are almost
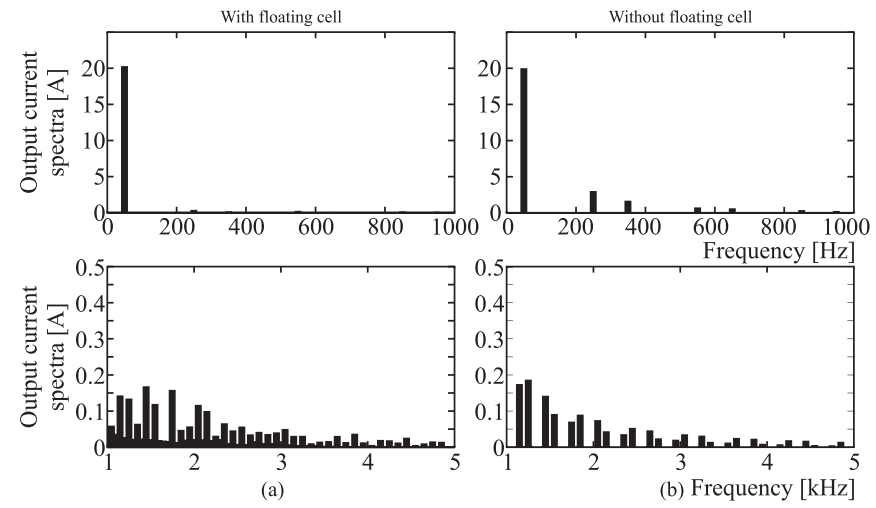

Fig. 6. Output current spectra: (a) With the floating cell; (b) Without the floating cell.

completely eliminated, while the spectra at high frequencies are comparable. Note that, as said before, the spectrum due to the MPC scheme is more spread in high frequency, than the spectrum without the floating cell, however most of those harmonics have a magnitude lower than $1 \%$.

\section{B. Start-up}

Fig. 7 shows the start-up performance of the proposed strategy. Fig. 7(a) shows the output current which, at the beginning, presents a high distortion due to the floating capacitor are discharged. As the floating dc-link voltages are increased (Fig. 7(d)), the current waveforms are improved. Notice also that during transient the currents slightly decrease their magnitudes. The only way to increase the capacitor voltages is to transfer active power from the main cells to them. Thus, when capacitor voltages are far away from their references, the FCS-MPC scheme decides to transfer active power to the capacitors (and reduce the power to the load), in order to accomplish with the quality function requirements.

\section{Output Current Step}

Finally, Fig. 8 shows the system behavior under a current magnitude and frequency step from $24 \mathrm{~A} / 50 \mathrm{~Hz}$ to $18 \mathrm{~A} / 30 \mathrm{~Hz}$. As can be seen in Fig. 8(a) the currents keep their quality. Moreover, neither significant transients nor oscillations are observed. This is also valid for the load and inverter voltages shown in Fig. 8(b) and (c) respectively. The floating capacitor voltages keep their average values, only the ripple decreases as the current reference magnitude does it.

\section{CONCLUSIONS}

This work shows the implementation of the FCS-MPC scheme for the control of the output currents and the floating voltages of an asymmetric CHB converter. This scheme allows high quality currents, good dc-voltage regulation and an excellent dynamic behavior. An additional advantage of the proposed technique is its conceptual simplicity which allows an easy interpretation of all the related variables.

The proposed control scheme guarantees the low switching frequency of the main cells, which provides the active power, 

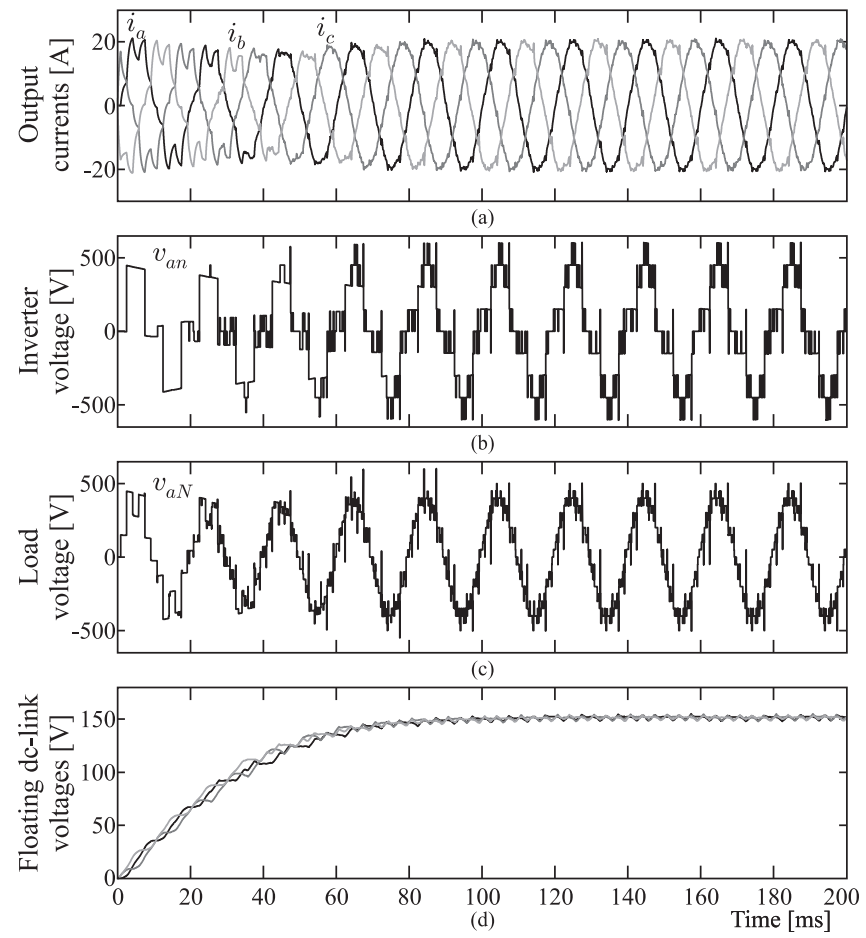

Fig. 7. Converter waveforms at the start-up.
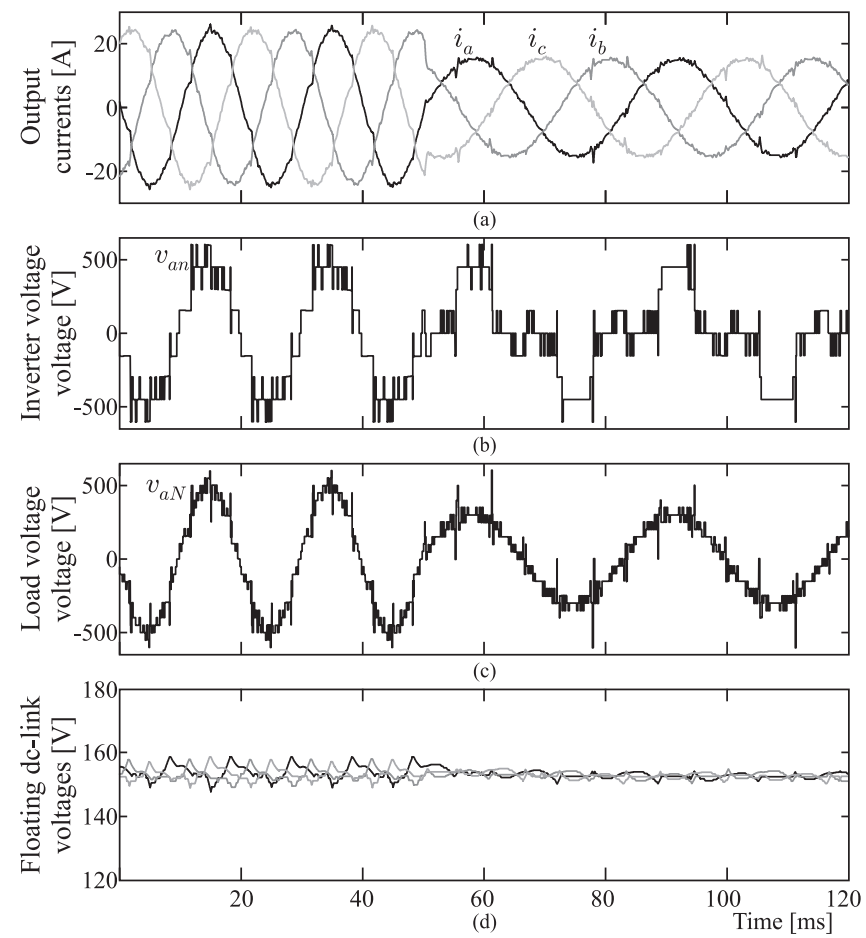

Fig. 8. Converter waveforms for a magnitude and frequency step, from $24 \mathrm{~A}-50 \mathrm{~Hz}$ to $18 \mathrm{~A}-30 \mathrm{~Hz}$.

while the floating cells commutate with a high frequency pattern, according with the minimization criteria imposed by the FCS-MPC algorithm. This method can be easily extended to more complex patterns for the main cell (additional commutation angles), this should lead to better quality signals but higher losses, then a tradeoff is established.

\section{ACKNOWLEDGMENTS}

The authors acknowledge the support of the Chilean Research Council (CONICYT), under grant FONDECYT grant 1085111.

\section{REFERENCES}

[1] J. Rodríguez, J. Lai and F. Z.Peng, "Multilevel Inverters: A Survey of Topologies, Controls, and Applications," IEEE Transactions on Industrial Electronics, vol. 49, 2002.

[2] L. G. Franquelo, J. Rodríguez, J. I. Leon, S. Kouro, R. Portillo, and M. A. M. Prats, "The age of multilevel converters arrives," IEEE Industrial Electronics Magazine, vol. 2, no. 2, pp. 28-39, Jun. 2008.

[3] S. Alepuz, S. Busquets-Monge, J. Bordonau, J. Gago, D. Gonzalez, and J. Balcells, "Interfacing renewable energy sources to the utility grid using a three-level inverter," IEEE Transactions on Industrial Electronics, vol. 53, no. 5, pp. 1504-1511, Oct. 2006.

[4] X. Yuan, W. Merk, J. Allmeling, "Stationary-Frame Generalized Integrators for Current Control of Active Power Filters With Zero SteadyState Error for Current Harmonics of Concern Under Unbalanced and Distorted Operating Conditions," IEEE Transactions on Industry Applications, vol. 38, no. 2, pp. 523-532, March/April 2002

[5] A. Lega, S. Munk-Nielsen, F. Blaabjerg, and D. Casadei, "Multilevel converters for ups applications: comparison and implementation." in Power Electronics and Applications, 2007 European Conference on, Sept. 2007, pp. 1-9.

[6] M. D. Manjrekar, T. A. Lipo, "Hybrid topology for multilevel power conversion." U.S. Patent, no. 6005788, December 1999.

[7] C. Rech and J. R. Pinheiro, "Hybrid multilevel converters: Unified analysis and design considerations," IEEE Transactions on Industrial Electronics, vol. 54, no. 2, pp. 1092-1104, Apr. 2007.

[8] Z. Du, L. Tolbert, J. Chiasson, and B. Ozpineci, "A cascade multilevel inverter using a single dc source," in Applied Power Electronics Conference and Exposition, 2006. APEC 'O6. Twenty-First Annual IEEE, March 2006, pp. 426-430.

[9] C. Silva, S. Kouro, J. Soto, and P. Lezana, "Control of an hybrid multilevel inverter for current waveform improvement," in Proc. IEEE International Symposium on Industrial Electronics ISIE 2008, June 30 2008-July 2 2008, pp. 2329-2335.

[10] S. Vazquez, J. Leon, L. Franquelo, J. Padilla, and J. Carrasco, "Dcvoltage-ratio control strategy for multilevel cascaded converters fed with a single dc source," Industrial Electronics, IEEE Transactions on, vol. 56, no. 7, pp. 2513-2521, July 2009.

[11] M. Veenstra and A. Rufer, "Control of a hybrid asymmetric multilevel inverter for competitive medium-voltage industrial drives," Industry Applications, IEEE Transactions on, vol. 41, no. 2, pp. 655-664, MarchApril 2005.

[12] J. Liao, K. Corzine, and M. Ferdowsi, "A new control method for singledc-source cascaded h-bridge multilevel converters using phase-shift modulation," in Applied Power Electronics Conference and Exposition, 2008. APEC 2008. Twenty-Third Annual IEEE, Feb. 2008, pp. 886-890.

[13] M. Marchesoni, M. Mazzucchelli, S. Tenconi, "A nonconventional power converter for plasma stabilization," IEEE Transactions on Power Electronics, vol. 5, no. 212-219, p. 2, April 1990.

[14] P. W. Hammond, "A new approach to enhance power quality for medium voltage drives," IEEE Transactions on Industry Applications, vol. 33 no. 1, pp. 202-208, Jan/Feb 1997.

[15] S. Mariethoz and A. Rufer, "Design and control of asymmetrical multilevel inverters," in Proc. IEEE 2002 28th Annual Conference of the] IECON 02 [Industrial Electronics Society, vol. 1, 5-8 Nov. 2002, pp. $840-845$

[16] G. C. Goodwin, M. M. Seron, and J. A. De Doná, Constrained Control \& Estimation: An Optimization Approach. Springer-Verlag, 2005.

[17] P. Cortés, M. P. Kazmierkowski, R. M. Kennel, D. E. Quevedo, and J. Rodríguez, "Predictive control in power electronics and drives," Industrial Electronics, IEEE Transactions on, vol. 55, no. 12, pp. 4312 4324, Dec. 2008.

[18] S. Kouro, P. Cortés, R. Vargas, U. Ammann, and J. Rodríguez, "Model predictive control: A simple and powerful method to control power converters," Industrial Electronics, IEEE Transactions on, vol. 56, no. 6, pp. 1826-1838, June 2009 